\title{
ARTICLE
}

\section{Direct Monte Carlo Dose Calculation Using Polygon-surface Computational Human Model}

\author{
Jong Hwi JEONG ${ }^{1}$, Chan Hyeong $\mathrm{KIM}^{1 *}$, Yeon Su YEOM ${ }^{1}$, Sungkoo $\mathrm{CHO}^{1}$, Min Suk Chung ${ }^{2}$, Kun-Woo $\mathrm{CHO}^{3}$ \\ ${ }^{1}$ Department of Nuclear Engineering, Hanyang University, 17 Haengdang-dong, Seongdong-gu, Seoul 133-791, Korea \\ ${ }^{2}$ Department of Anatomy, Ajou University School of Medicine, San 5 Wonchon-dong, Yeongtong-gu, Suwon 443-749, Korea \\ ${ }^{3}$ Korea Institute of Nuclear Safety, 19 Guseong-dong, Yuseong-gu, Daejeon, 305-600, Korea
}

\begin{abstract}
In the present study, a voxel-type computational human model was converted to a polygon-surface model, after which it was imported directly to the Geant 4 code without using a voxelization process, that is, without converting back to a voxel model. The original voxel model was also imported to the Geant 4 code, in order to compare the calculated dose values and the computational speed. The average polygon size of the polygon-surface model was $\sim 0.5$ $\mathrm{cm}^{2}$, whereas the voxel resolution of the voxel model was $1.981 \times 1.981 \times 2.0854 \mathrm{~mm}^{3}$. The results showed a good agreement between the calculated dose values of the two models. The polygon-surface model was, however, slower than the voxel model by a factor of 6-9 for the photon energies and irradiation geometries considered in the present study, which nonetheless is considered acceptable, considering that direct use of the polygon-surface model does not require a separate voxelization process..
\end{abstract}

\section{KEYWORDS: polygon-surface model, voxel model, dose calculation, G4tesselatedSolid, Geant4}

\section{Introduction ${ }^{1}$}

Two different kinds of computational human models are currently available for Monte Carlo dose calculations: stylized models based on mathematical surfaces, and voxel models based on tomographic image ${ }^{1,2)}$. Recently, investigators have developed hybrid-type human models by converting their voxel models to polygon or non-uniform rational B-spline (NURBS) surface models ${ }^{3-6)}$. These surface models have both the flexibility of the stylized models and the realism of the voxel models. Lee et al. ${ }^{5}$ converted a newborn voxel model to a NURBS surface model and then changed the posture of the model. Additionally, a male newborn model was created from a female newborn model by replacing the genital organs. Xu et al. ${ }^{6}$ developed three pregnant female models representing different gestational periods for one person, using polygon and NURBS surfaces. These innovative investigations would be very difficult or impossible with the original voxel models.

The surface model, however, has a critical limitation, in that it has to be converted back to a voxel model to be used in Monte Carlo dose calculations ${ }^{5,6}$. This so-called "voxelization" process eliminates the principal advantages of the surface model, which are the capability of modeling very thin structures and the capability of 4-D Monte Carlo simulation.

In the present study, a high-quality voxel model, HDRK-Man ${ }^{7}$, was converted to a polygon-surface model, after which it was imported directly to the Geant $4 \operatorname{code}^{8)}$ without any voxelization process. The polygon-surface model in the Geant 4 code was then used to calculated organ doses and, thereby, a weighted dose, which is very similar to

*Corresponding Author, Tel. +82-2-2220-0513, Fax. +82-2-2220-4059, E-mail:chkim@hanyang.ac.kr effective dose, for external photon beams of different directions and energies. The results were finally compared with those of the original HDRK-Man voxel model.

\section{Methods}

The HDRK-Man voxel model, which was constructed in Korea using high-resolution color photographic images obtained by serial sectioning of a cadaver of a Korean adult male ${ }^{9)}$, was converted to a polygon-surface model. The skin and the other organs of the HDRK-Man model, except for the skeletal system and intestines, were directly converted to polygon-surfaces using the 'surface rendering' function in 3D-DOCTOR ${ }^{\mathrm{TM}}$ (Able Software Corp., Lexington, MA).

The primary polygon surfaces generated by the surface rendering function, however, cannot be used directly in a Monte Carlo simulation code because they include many imperfections such as rough surfaces, abnormal faces, and holes. These imperfections therefore were removed before the polygon surfaces were imported to the Geant 4 code. The number of the polygons also was optimized for each organ by repeatedly using the 'decimate' function through the refinement process, which function reduces the number of the polygons while maintaining the original shapes of the organs.

The conversion and refinement of the polygon surfaces were mostly automatic processes for most of the organs, excepting the skull and intestines, for which manual modifications were used intensively. It was not possible to construct polygon-surface models using the HDRK-Man voxel model for very complicated organs, that is, the skeletal system and intestines, for which computed tomography (CT) image data from the cadaver and a separate set of high-resolution $(\sim 1 \mathrm{~mm}$ $\mathrm{x} 1 \mathrm{~mm} \times 1 \mathrm{~mm}$ ) voxel data were used, respectively.

With regard to the intestines, it was difficult, given their complexities and tortuous shapes, to convert even the 
high-resolution voxel models to polygon surfaces without significant intersections. In this case, therefore, the voxel models were first converted to primitive polygon surfaces, which were then used to generate the contours of the intestines along the paths of the intestines. The NURBS surfaces of the intestines were then generated by linking the contours with the 'Loft' function in Rhinoceros ${ }^{\mathbb{R}} 4.0$ (McNeel North America, Seattle, WA). Those surfaces finally were converted back to polygon surfaces. There were some minor distortions in the shapes of the polygon models due to the use of multiple conversion steps, but those distortions were not significant and, as such, were ignored in the present study. The polygon surfaces constructed were only for the exterior surfaces of the intestines; the interior surfaces of the intestine walls were generated by defining the thicknesses of the intestine walls in consideration of the intestinal masses.

In order to import the HDRK-Man polygon-surface model to the Geant4 code, the G4TessellatedSolid class was used, which was originally developed for importing computer-aided design (CAD) models. If the polygon-surface object of the G4TessellatedSolid class is not fully enclosed or includes abnormal surfaces, the Geant 4 code is terminated, displaying an error message indicating that it cannot determine whether a given point is inside or outside of a volume. Therefore, only after confirming that there was no imperfection or interference, the vertex coordinates of the individual triangular faces were exported, with Rhinoceros ${ }^{\circledR} 4.0$, to external data files, which were then read by the G4TessellatedSolid class.

\section{Results and Discussion}

A total of fifteen organs in the original HDRK-Man voxel model were converted to polygon-surface models, as shown in Table 1. The organs listed in Table 1 are the organs that are used to determine the effective dose ${ }^{10)}$. The principal organs with their specific tissue weighting factors were all converted to the polygon-surface models, except for the red bone marrow (RBM), for which a polygon surface is inappropriate. The dose to the RBM, however, can be estimated by calculating the detailed dose distribution in the entire skeletal system. The organs included in the 'remainder tissue' were not converted, except for the kidneys and small intestines.

The polygon-surface model, a collection of organ models, was successfully imported to the Geant4 code with the G4TessellatedSolid class for Monte Carlo dose calculations. The original HDRK-Man voxel model also was imported to the Geant4 code, using the G4VNestedParameterisation class. The dose calculations were performed with a personal computer having a $2.40 \mathrm{GHz}$ Intel Core ${ }^{\mathrm{TM}} 2$ Quad Processor Q6600 and 2 GB RAM. Figure 1 shows the original HDRK-Man voxel model and the polygon-surface model as modeled in the Geant 4 code. The average facet size of the polygon-surface model was $\sim 0.5 \mathrm{~cm}^{2}$, whereas the voxel resolution of the voxel model was $1.981 \times 1.981 \times 2.0854 \mathrm{~mm}^{3}$.

The organs doses were calculated by transporting photons and resulting secondary electrons using the standard electromagnetic (EM) package of Geant4.9.1.p02 ${ }^{8)}$. The irradiation geometries considered in the present study were
Table 1 HDRK-Man polygon-surface model

\begin{tabular}{|c|c|c|c|}
\hline Organ/Tissue & $\begin{array}{c}\text { Tissue } \\
\text { weighting } \\
\text { factor } \mathrm{w}_{\mathrm{T}} \\
\text { (ICRP 103) }\end{array}$ & $\begin{array}{c}\text { Mass } \\
(\mathrm{kg})\end{array}$ & $\begin{array}{c}\text { Number } \\
\text { of } \\
\text { polygons }\end{array}$ \\
\hline Colon & 0.12 & 323.515 & 10068 \\
\hline Lung & 0.12 & 1122.974 & 5334 \\
\hline Stomach & 0.12 & 138.930 & 2000 \\
\hline Breast & 0.12 & 22.147 & 2000 \\
\hline Bone marrow (red) & 0.12 & - & - \\
\hline Gonads (testis) & 0.08 & 28.815 & 498 \\
\hline Bladder & 0.04 & 38.913 & 1366 \\
\hline Esophagus & 0.04 & 40.203 & 2000 \\
\hline Liver & 0.04 & 1438.690 & 2688 \\
\hline Thyroid & 0.04 & 15.017 & 1000 \\
\hline Bone surface & 0.01 & 9894.154 & 38950 \\
\hline Brain & 0.01 & 1516.740 & 7492 \\
\hline Salivary glands & 0.01 & 81.398 & 3000 \\
\hline Skin & 0.01 & 2395.413 & 29998 \\
\hline Remainder tissue & 0.12 & & \\
\hline Adrenals & & - & - \\
\hline ET region & & - & - \\
\hline Gall bladder & & - & - \\
\hline Heart & & - & - \\
\hline Kidneys & & 338.504 & 2338 \\
\hline Lymphatic nodes & & - & - \\
\hline Muscle & & - & - \\
\hline Oral mucosa & & - & - \\
\hline Pancreas & & - & - \\
\hline Prostate & & - & - \\
\hline Small intestine & & 372.544 & 7500 \\
\hline Spleen & & - & - \\
\hline Thymus & & - & - \\
\hline
\end{tabular}

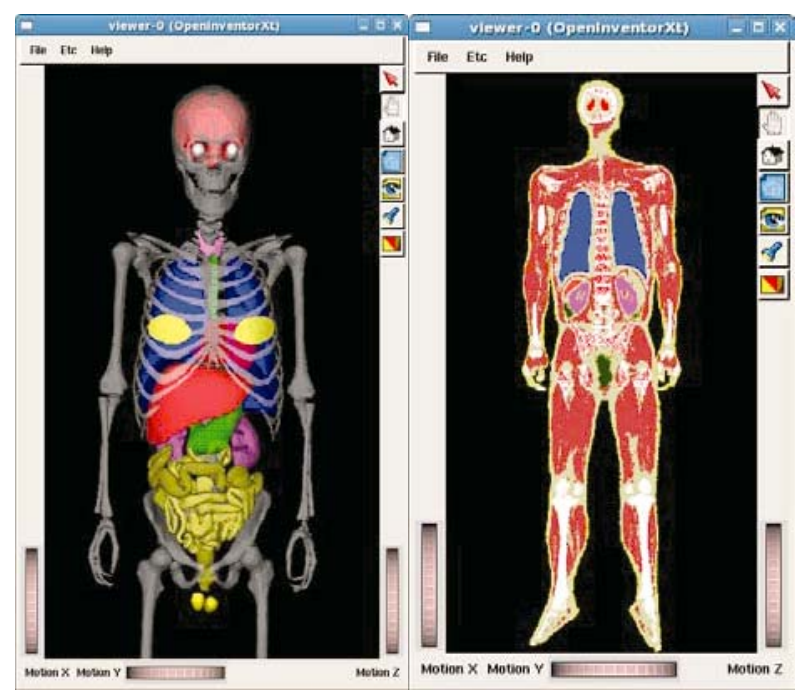

Fig. 1 HDRK-Man polygon model (left) and voxel model (right) as imported to Geant4 

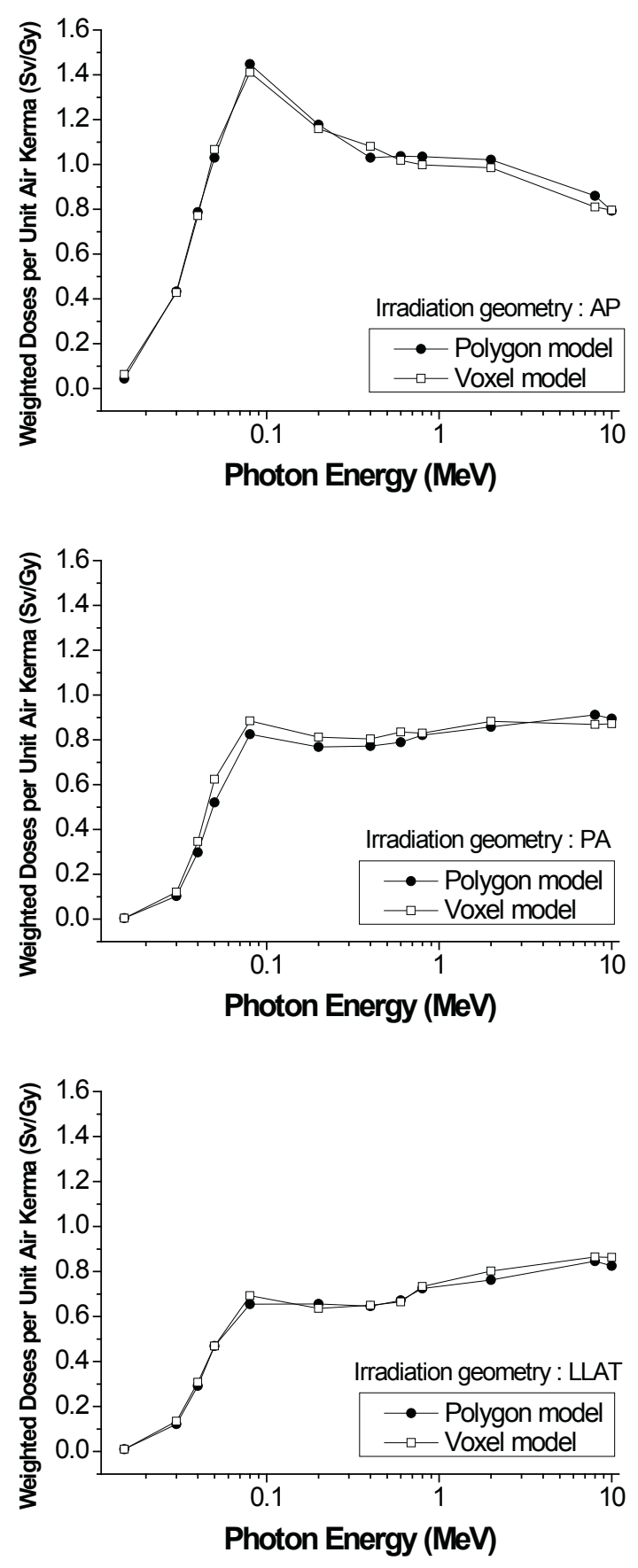

Fig. 2 Dose conversion coefficients (DCC) for weighted dose calculated by polygon-surface and voxel models for anterior-posterior (AP), posterior-anterior (PA) and left-lateral (LLAT) irradiation geometries

broad parallel photon beams of anterior-posterior (AP), posterior-anterior (PA), and left-lateral (LLAT) directions. The considered photon energies were $0.015-10 \mathrm{MeV}$ for each of the irradiation geometries. The G4PSEnergyDeposit scorer class was used to score the deposited energies in the organs and, thereby, the organ doses (DT), which were then used to determine a "weighted dose" based on the tissue weighting
Table 2 Computation times of polygon-surface and original voxel models for anterior-posterior (AP) irradiation geometry

\begin{tabular}{ccc}
\hline $\begin{array}{c}\text { Energy } \\
(\mathrm{MeV})\end{array}$ & $\begin{array}{c}\text { Polygon model } \\
(\mathrm{mm}: \mathrm{ss})\end{array}$ & $\begin{array}{c}\text { Voxel model } \\
(\mathrm{mm}: \mathrm{ss})\end{array}$ \\
\hline 0.015 & $120: 01$ & $20: 30$ \\
0.030 & $196: 19$ & $27: 39$ \\
0.040 & $249: 10$ & $32: 03$ \\
0.050 & $291: 59$ & $35: 48$ \\
0.080 & $360: 52$ & $41: 28$ \\
0.200 & $396: 11$ & $44: 48$ \\
0.400 & $379: 56$ & $44: 52$ \\
0.600 & $368: 10$ & $44: 35$ \\
0.800 & $358: 55$ & $44: 05$ \\
2.000 & $336: 40$ & $41: 39$ \\
8.000 & $351: 22$ & $41: 27$ \\
10.00 & $362: 20$ & $41: 54$ \\
\hline
\end{tabular}

factors given for the effective dose ${ }^{10)}$ (See also Table 1). The weighted dose is not the effective dose but rather a quantity conceptually very close to it. The calculated weighted dose values finally were divided by the air kerma values determined for the same irradiation geometries, in order to calculate the dose conversion coefficient (DCC) values for the weighted dose.

Figure 2 compares the DCC values of the polygon-surface model with those of the original HDRK-Man voxel model, showing a good agreement between the two models. The difference was less than $7 \%$ for photon energies $\geq 0.03 \mathrm{MeV}$, which is negligible considering the difference in the model geometries (voxel vs. polygon-surface) and the statistical fluctuations.

There was, however, a significant difference in the computation time between the two models. Table 2 illustrates the comparison, showing that the computation time of the polygon model is about 6-9 times longer than that of the voxel model. It is acknowledged that a direct comparison of these two different types of models for computation speed has very limited meaning, but the comparison provides at least a general idea on the speed of a polygon-surface model in comparison with a voxel model. The difference in computation time was significant, but considered acceptable nonetheless, considering that direct use of the polygon-surface model does not require a separate voxelization process. The difference in computation time moreover will decrease as the Geant4 code is optimized to polygon surfaces.

\section{Conclusions}

A voxel-type computational human model, HDRK-Man, was converted to a polygon-surface model, after which it was imported directly to the Geant 4 code for Monte Carlo dose calculations. The results were then compared with the values of the original HDRK-Man voxel model, showing a good agreement. The polygon-surface model was, however, slower than the voxel model by a factor of 6-9 for the same 
irradiation geometries considered in the present study, which nonetheless is considered acceptable, considering that direct use of the polygon-surface model does not require a separate voxelization process.

\section{Acknowledgment}

This work was supported by the Nuclear R\&D Program in Korea through KINS and BAERI. This work was also supported by Korean Ministry of Knowledge Economy (2008-P-EP-HM-E-06-0000)/Sunkwang Atomic Energy Safety Co., Ltd.

\section{References}

1) C. Lee, J. Lee, "Computational Anthropomorphic Phantoms for Radiation Protection Dosimetry: Evolution and Prospects," Nucl. Eng. Technol., 38[3], 239-250 (2006).

2) H. Zaidi, X. G. Xu, "Computational Anthropomorphic Models of the Human Anatomy: The Path to Realistic Monte Carlo Modeling in Radiological Sciences," Annu. Rev. Biomed. Eng., 9, 471-200 (2007)

3) W. P. Segars, B. M. W. Tsui, E. C. Frey, G. A. Johnson, S. S. Berr., "Development of a 4D Digital Mouse Phantom for Molecular Imaging Research," Mol. Imaging Biol., 6[3], 149-159 (2004).

4) C. Lee, C Lee, D. Lodwick, W. E. Bolch, "NURBS-based 3-d
Anthropomorphic Computational Phantoms for Radiation Dosimetry Applications," Radiat. Prot. Dosim., 127[1-4], 277-232 (2007).

5) C. Lee, D. Lodwick, D. Hasenauer, J. L. Williams, C. Lee, W. E. Bolch, "Hybrid Computational Phantoms of the Male and Female Newborn Patient: NURBS-based Whole-body Models," Phys. Med. Biol., 52[12], 3309-3333 (2007).

6) $\mathrm{X}$. G. Xu, V. Taranenko, J. Zhang, C. Shi, "A Boundary-Representation Method for Designing Whole-Body Radiation Dosimetry Models: Pregnant Females at the Ends of Three Gestational Periods-RPI-P3, -P6 and -P9," Phys. Med. Biol., 52[23], 7023-7044 (2007).

7) C. H. Kim, S. H. Choi, J. H. Jeong, C. Lee, M. S. Chung, "HDRK-Man: a Whole-Body Voxel Model based on High-Resolution Color Slice Images of a Korean Adult Male Cadaver," Phys. Med. Biol., 53[15], 4093-4106 (2008).

8) S. Agostinelli, et al., "GEANT4-A Simulation Toolkit," Nucl. Instrum. Methods Phys. Res., Sect. A, 506[3], 250-303 (2003).

9) J. S. Park, M. S. Chung, J. Y. Kim, H. S. Park, "Visible Korean Human: Another trial for making serially sectioned images," Medical Imaging, 4681[3], 171-183 (2002).

10) International Commission on Radiological Protection, The 2007 recommendations of the International Commission on Radiological Protection, J. Valentin, Elsevier Publishing, New York, 173-213 (2008). 